\title{
NIRS-Based Hyperscanning Reveals Inter-brain Neural Synchronization during Cooperative Jenga Game with Face-to-Face Communication
}

\author{
Ning Liu ${ }^{1 *}$, Charis Mok ${ }^{2}$, Emily E. Witt ${ }^{2}$, Anjali H. Pradhan ${ }^{3}$, Jingyuan E. Chen ${ }^{4,5}$ \\ and Allan L. Reiss ${ }^{1,4}$ \\ ${ }^{1}$ Center for Interdisciplinary Brain Sciences Research, Department of Psychiatry and Behavioral Sciences, School of \\ Medicine, Stanford University, Stanford, CA, USA, ${ }^{2}$ Program in Human Biology, Stanford University, Stanford, CA, USA, \\ ${ }^{3}$ Department of Molecular and Cell Biology, University of California, Berkeley, CA, USA, ${ }^{4}$ Department of Radiology, Stanford \\ University, Stanford, CA, USA, ${ }^{5}$ Department of Electrical Engineering, Stanford University, Stanford, CA, USA
}

OPEN ACCESS

Edited by:

Nobuo Masataka,

Kyoto University, Japan

Reviewed by:

Tao Liu,

Zhejiang University, China

Hirokazu Doi,

Nagasaki University, Japan

${ }^{*}$ Correspondence:

Ning Liu

ning/@stanford.edu

Received: 15 December 2015 Accepted: 18 February 2016 Published: 08 March 2016

Citation:

Liu N, Mok C, Witt EE, Pradhan AH, Chen JE and Reiss AL (2016) NIRS-Based Hyperscanning Reveals Inter-brain Neural Synchronization during Cooperative Jenga Game with

Face-to-Face Communication.

Front. Hum. Neurosci. 10:82.

doi: 10.3389/fnhum.2016.00082
Functional near-infrared spectroscopy (fNIRS) is an increasingly popular technology for studying social cognition. In particular, fNIRS permits simultaneous measurement of hemodynamic activity in two or more individuals interacting in a naturalistic setting. Here, we used fNIRS hyperscanning to study social cognition and communication in human dyads engaged in cooperative and obstructive interaction while they played the game of Jenga ${ }^{\mathrm{TM}}$. Novel methods were developed to identify synchronized channels for each dyad and a structural node-based spatial registration approach was utilized for interdyad analyses. Strong inter-brain neural synchrony (INS) was observed in the posterior region of the right middle and superior frontal gyrus, in particular Brodmann area 8 (BA8), during cooperative and obstructive interaction. This synchrony was not observed during the parallel game play condition and the dialog section, suggesting that BA8 was involved in goal-oriented social interaction such as complex interactive movements and social decision-making. INS was also observed in the dorsomedial prefrontal cortex (dmPFC), in particular Brodmann 9, during cooperative interaction only. These additional findings suggest that BA9 may be particularly engaged when theory-of-mind (ToM) is required for cooperative social interaction. The new methods described here have the potential to significantly extend fNIRS applications to social cognitive research.

Keywords: functional near-infrared spectroscopy, fNIRS, hyperscanning, cooperation, obstructive interaction, inter-brain neural synchronization (INS)

\section{INTRODUCTION}

A pervasive challenge in social neuroscience is how to model everyday human interactions in the laboratory (Hari and Kujala, 2009). The technique of hyperscanning has enormous potential to address this problem by enabling simultaneous recording of brain function in multiple interacting subjects (Babiloni and Astolfi, 2014). Hyperscanning paradigms provide a valuable platform for observing neural signatures of social cognition during social interaction. The first hyperscanning study can be traced back to an electroencephalography (EEG) study in 1965 (Duane and Behrendt, 1965). More recently, researchers have applied this 
technique with a variety of neuroimaging modalities, including functional magnetic resonance imaging (fMRI; Montague et al., 2002), functional near-infrared spectroscopy (fNIRS; Funane et al., 2011; Cui et al., 2012), magnetoencephalography (MEG; Baess et al., 2012) and EEG (Babiloni et al., 2011; Kawasaki et al., 2013). NIRS-based hyperscanning is a technique in which single or multiple instruments are used for simultaneous measurement of brain activity in two or more people (Scholkmann et al., 2013; Balconi and Molteni, 2015). Compared with other functional imaging techniques, fNIRS hyperscanning has the advantage of being cost-effective, portable, and more tolerant to movement. Furthermore, fNIRS allows measurement of brain activity in environments with greater ecological validity, an especially important advantage, as studies have shown that simulated tasks do not always generate the same brain activity as they do in their real-life settings (Okamoto et al., 2004). As such NIRS is increasingly considered an emerging tool in social cognitive neuroscience that can be used in a natural context with free hand movement and face-to-face oral communication. In particular, this technology offers an easy to apply and reliable brain imaging technology for measuring inter-personal interactions.

NIRS-based hyperscanning has been employed by a number of researchers to investigate interactive social behavior. For instance, Cui et al. (2012) studied the interpersonal coherence in the superior frontal cortex between two players while they played a computer-based cooperation game side by side; Holper et al. (2012) investigated the between-brain connectivity of premotor cortices in paired subjects during imitation performance of a paced finger-tapping task; the same group of people also showed a significant increase in between-brain coherence during joint n-back task performance as compared to a baseline condition (Dommer et al., 2012); Jiang et al. (2012) studied the left frontal cortices of paired participants during face-to-face communication, and showed a significant increase in the neural synchronization in the left inferior frontal cortex during face-toface dialog between partners; the same group also investigated interpersonal neural synchronization in the left temporo-parietal junction during a group discussion among triads, and showed that the inter-brain neural synchrony (INS) for the leaderfollower pairs was higher than that for the follower-follower pairs (Jiang et al., 2015); Liu et al. (2015c) studied the right inferior frontal gyrus of paired participants as they played a turn-taking (game builder or partner) game. The builder in the cooperation condition showed higher activation than his/her partner, though the same builder in the competition condition showed lower activation than in the cooperation condition. In many of these studies, interpersonal or INS has been used to denote the Wavelet Transform Coherence (WTC) of measured fNIRS signals between paired participants, in most cases, using oxy-hemoglobin concentration changes.

An important issue in fNIRS hyperscanning concerns how to spatially register cortical location data in dyadic and group analyses. A conventional way to determine the spatial location of an fNIRS measuring channel is to follow the 10-20 system, which requires an accurate placement of optodes across subjects. To date, fNIRS hyperscanning studies have solely used channel-wise analysis, which assumes that the location of each channel across subjects is representative of a common anatomical location. This approach assumes that using identical channels for both interbrain and group analyses is methodologically valid. However, this is often not the case when placing fNIRS probes on the heads of individual subjects. Although, no single method has been broadly accepted to address this issue in hyperscanning studies, several approaches have been described for fNIRS data spatial registration of group data (Tsuzuki and Dan, 2014). For example, one suggestion is to implement image reconstruction algorithms based on the solution of the inverse problem for light transport, and to map fNIRS data to a three-dimensional voxel space, allowing voxel-wise analytic approaches (Tsuzuki et al., 2012). However, this method is primarily intended for use with a high-density fNIRS system and requires an MRI image for each subject. Another approach is to identify a ROI based on an anatomical atlas (Okamoto et al., 2009; Yanagisawa et al., 2010). In this approach, center coordinates are designated and neighboring channels or voxels are extracted to represent the functional status of a ROI. However, as Tsuzuki and Dan point out (Tsuzuki and Dan, 2014), setting a ROI by integrating channels or voxels is equivalent to spatial smoothing, and thus different ROI sizes result in different degrees of spatial filtering.

The study presented here was designed to investigate neural synchrony using fNIRS in a naturalistic, interactive setting during which subjects are able to orally communicate face-toface while playing a non-computerized game, Hasbro's Jenga ${ }^{\mathrm{Tn}}$. Jenga has been used in various studies in social psychology, such as friendship (Wright et al., 2002; Page-Gould et al., 2008), the study of social-interactive behavior of children with social deficits (Kawaguchi et al., 2010), and psychoeducation (Briggs et al., 2011). In our study, we created conditions for the interacting dyads that could be implemented in a naturalistic manner. Three different levels of cooperation were used: full cooperation, parallel game play, and obstructive interaction. As consistent with previous descriptions (OPTIC, Paulson, 1974), full cooperation incorporates the highest level of interpersonal cooperation, while obstructive interaction is considered to be least conducive for cooperation. Previous hyperscanning studies of turn-based interdependent behaviors (Liu and Pelowski, 2014) have shown inter-brain synchronization during both cooperative and competitive interactions (Jiang et al., 2015; Liu et al., 2015b), but not in independent play (Cui et al., 2012). Thus, we hypothesized that we would observe inter-brain neural synchrony (INS) during both cooperation and obstructive interaction conditions, but not during parallel play condition. For the imaging data analysis, instead of using channel-wise analysis, we developed a new method to identify synchronized channels within a dyad and a structural node-based spatial registration approach for inter-dyad analyses.

\section{MATERIALS AND METHODS}

\section{Participants}

Eighteen healthy volunteers (age $21.1 \pm 1.7$ years) participated in the study. Standardized phone interviews were conducted 
to screen for handedness (Edinburgh Handedness Inventory) and medical/psychiatric history. None of the subjects that participated in the study reported a history of significant medical, neurologic or psychiatric illness. All subjects were right-handed college students, and were already familiar with the game of Jenga ${ }^{\mathrm{m}}$. Unacquainted subjects were formed into nine pairs (female/female, $n=2$; male/male, $n=2$; and male/female, $n=5$ ). The study protocol was approved by the Stanford University Institutional Review Board. Written informed consent was obtained from all subjects.

\section{Task}

The task was comprised of three experimental conditions: cooperation, parallel play, obstructive interaction and the control section: dialog. The dialog section was designed to control for neural activation resulting from oral communication. The order of the three task conditions was counterbalanced across dyads. During the cooperation, parallel play, and obstructive interaction conditions, participant dyads played the game of Jenga according to different objectives.

In a conventional Jenga game, players remove wooden blocks from a stacked tower formation and place it on the top of the tower. Moving the wooden blocks from the lower portion of the tower to the top will make the tower less stable. The conventional goal of the game is to keep the tower standing without falling. In our study, we introduced additional rules to structure the cooperation and obstructive interaction during the game. The general game rule for the cooperation and obstructive interaction conditions was that players took turns removing a single wooden block from a single stacked tower followed by placing the block on the top of the tower. Each player was allowed to touch and move only one block during his or her turn.

The specific rules for each experimental conditions and the dialog section are further described as follows:

(1) During the cooperation condition, dyads were instructed to work together to build the tallest possible tower in the time allotted. They took turns making a move, and were instructed to talk to one another about strategy and agree on which block to move before they made any physical movement of the wooden block. For instance, player 1 would be the player in-turn in a trial. Player 1 might propose moving a middle block on the third floor, while player 2 would advise that moving the middle block on the fifth floor would be better. The two players would then need to discuss and agree on a specific block and movement before player 1 could make any movement.

(2) During the obstructive interaction condition, dyads were instructed to adopt a strategy with their partner in order to increase the likelihood that the tower would fall. As in the cooperation condition, subjects took turns making a move, and were asked to talk about each move; however they did not have to provide truthful advice, nor listen to their partner's recommendations. For instance, player 2 would be the player in-turn in a trial. Player 2 might propose moving a middle block on the fifth floor, while player 1 would suggest moving the left block on the third floor. Player 2 would surmise that moving the block on the third floor would make the tower fall, so player 2 would move the middle block on the fifth floor anyway without following the suggestion of player 1 .

(3) During the parallel play condition, subjects played Jenga in the same area, built separate towers, with the goal of building a tower without falling. During this condition they narrated their moves to themselves, explaining their strategy, but did not interact with each other. Thus, the subject's attention was focused primarily on his/her own materials.

(4) During the dialog section, dyads did not play Jenga, but mimicked task conversation by discussing a given topic and conversing in a back and forth manner with short sentences.

Each task condition was divided into two $120 \mathrm{~s}$ blocks that were separated by a $60 \mathrm{~s}$ dialog block. A $60 \mathrm{~s}$ rest period was used when switching between task conditions. There were also $60 \mathrm{~s}$ rest periods before the first task condition and after the last task condition that served as a baseline state. Figure 1A shows an example of the task design as follows: Rest, Obstructive block, Dialog, Obstructive block, Rest, Cooperation block, Dialog, Cooperation block, Rest, Parallel Play block, Dialog, Parallel Play block, Rest. The given dialog topic between the two cooperation blocks was to "Talk about your favorite foods." In between the two obstructive interaction blocks, the prompt was to "Talk about the courses you've taken." In between the two parallel play blocks, the prompt was to "Talk to yourself about your day."

\section{Experimental Procedure}

The experiment was conducted with the dyad and two research staff members in one room. One staff member operated the NIRS machine while the other introduced the task and experimental procedure. The two subjects comprising a dyad were seated comfortably on opposite sides of a table facing each other, with their forearms resting on the table to minimize excess arm and neck movement. During the cooperative and obstructive interaction conditions, the Jenga tower was placed in the center of the table so that each subject could see two faces of the tower and had a clear view of his or her partner's moves (Figure 1B). During the parallel play condition, a tower was placed in front of each subject. The tower was blocked from subjects' view during all rest periods and dialog blocks to prevent them from working on the tower during those periods. Before the task started, subjects listened to the examiner's verbal task instructions and watched video demonstrations of each condition. Additionally, subjects had a chance to practice the task to ensure they understood the instructions. Subjects were designated as " 1 " and " 2 " prior to the start of the experiment and subject 1 was instructed to make the first move every time a new condition began. Once the experiment started, subjects were not permitted to talk to the research staff members. After the task was complete, subjects completed a questionnaire to describe the strategies that used during the cooperative and obstructive interaction conditions to ensure they were following instructions. 


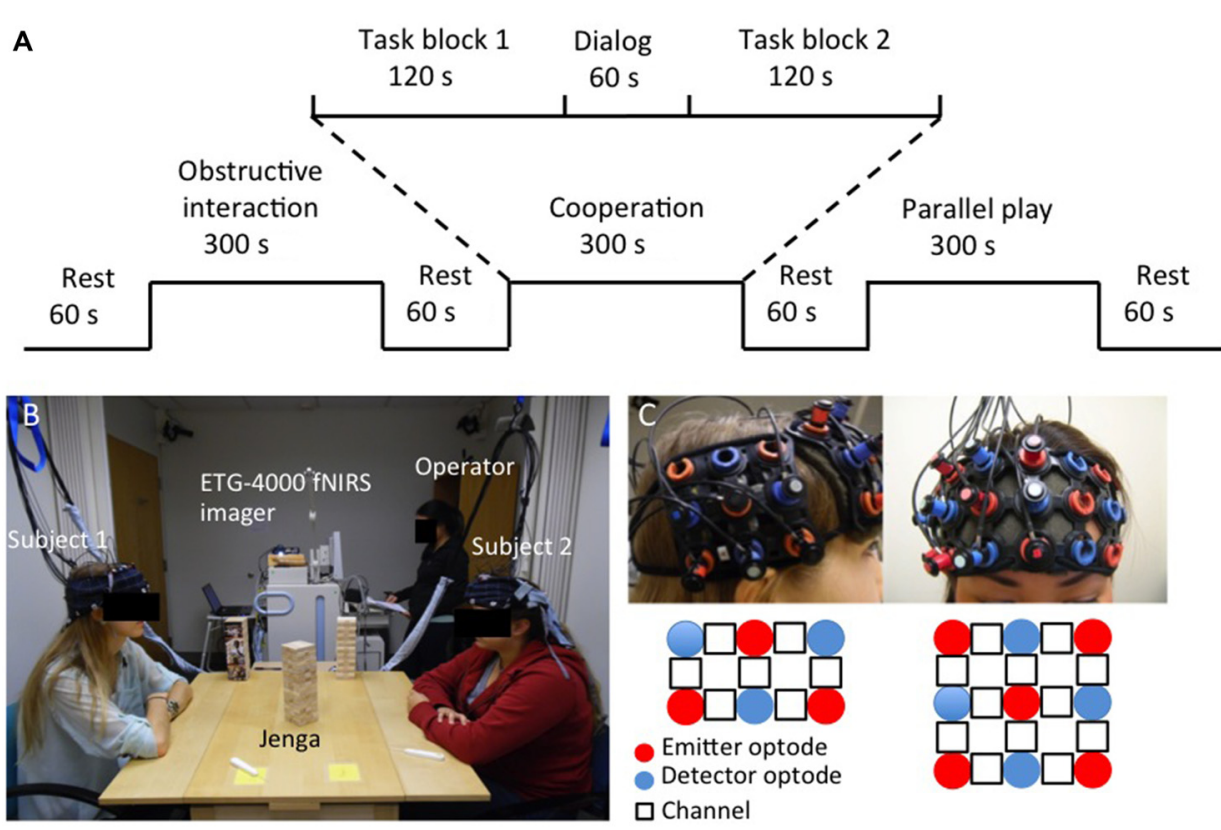

FIGURE 1 | Task design (A), experimental setup (B) and probe configuration (C).

\section{Videotaping and Data Coding}

For exploratory purposes, the performance of five dyads was fully videotaped for later data coding. A camcorder was place on a side table at 3 feet 6 inches above the floor, and 3 feet away from the game table. The recorder was turned on when the task started, and turned off when the entire experiment was complete.

After videotaping was completed, we determined the onset time for each subject to move the wooden blocks. Since each subject took turns to move the blocks, the social interaction frequency was determined as the inverse of the time interval between two continuous movements of the block.

\section{fNIRS Data Acquisition}

fNIRS signals were acquired in the two subjects simultaneously using an ETG-4000 Optical Topography system (Hitachi Medico Co., Tokyo, Japan) with a sampling rate of $10 \mathrm{~Hz}$. The measurement patches consisted of an evenly distributed array of alternating emitter and detector fiber bundles (optodes). Optodes were spaced $30 \mathrm{~mm}$ apart, resulting in a spatial resolution of $30 \mathrm{~mm}$ for the system. A channel represented the area measured by one emitter-detector pair, and the channel location was defined as the center position of the emitter-detector optodes. A single " $3 \times 3$ " measurement patch containing nine optodes was positioned over the right prefrontal cortex (rPFC) of each subject's head, resulting in 12 measurement channels. A single " $3 \times 2$ " measurement patch consisting of six optodes was positioned over the right superior temporal sulcus region (rSTS) of each subject's head, resulting in seven measurement channels (Figure 1C). We chose these two regions based on their previously identified roles in social cognitive processes.
Specifically, our previous study showed that increased coherence occurred in the rPFC of dyads during a cooperative computer task (Cui et al., 2012). Other game-based EEG studies also reported increased interbrain synchronization in the prefrontal cortex during cooperative decision-making (Astolfi et al., 2009). The rSTS has been implicated as a critical structure for processing socially relevant information, such as body or facial movement (Allison et al., 2000).

The patch placement was based on the 10-20 system. Specifically, the inside edge of the " $3 \times 3$ " patch was aligned to the midline (i.e., the arc running from the nasion through $\mathrm{Cz}$ to the inion), and the bottom row of the patch was right on top of the subjects' eyebrows. The inside edge of the " $3 \times 2$ " patch was placed above the right ear $\left(\mathrm{T}_{3}\right)$, and the bottom row was parallel to the floor and in line with $\mathrm{T}_{3}-\mathrm{T}_{5}$. Within participant pairs, patches were also examined and adjusted to ensure similarity of position based on the best judgment of the operator. The locations of the optodes on the head were obtained by a 3D magnetic digitizer system (PATRIOT, Polhemus, Colchester, VT, USA) right after the task was completed, which measured the placement of each optode in relation to five reference points on the subject's head (nasion, left and right preauricular points, vertex, and inion).

\section{Single Subject fNIRS Data Pre-Processing}

The recorded changes in optical density were first converted into concentration changes of oxy-hemoglobin and deoxyhemoglobin using the modified Beer-Lambert law (Delpy et al., 1988). An fNIRS channel was determined to be "noisy" if the heartbeat was not identifiable in the corresponding wavelet transform map (Liu et al., 2015a), and all noisy channels were 
excluded from subsequent analyses. The channel-wise individual fNIRS data was registered to a standard MRI brain template (MNI152). Specifically, the channel positions in Cartesian space were first converted to the Montreal Neurological Institute (MNI) standardized space (Singh et al., 2005), and then projected to the cortical surface (Cui et al., 2011). The obtained MNI coordinates at the cortical surface can either be plotted in a 3-D space (using the plot3 command in MATLAB), or on a rendered brain template (using xjView toolbox ${ }^{1}$ ). For representation purposes, after projecting the channel position to the cortical surface, we drew a spherical region around the projection point with a radius of five voxels $(\sim 17.5 \mathrm{~mm})$, and removed any portion of the sphere that fell outside the brain mask, only keeping the voxels inside the brain (Cui et al., 2011). This way, we could signify the spatial resolution of the system $(3 \mathrm{~cm})$.

\section{Paired Subjects fNIRS Data Analysis}

Although attempts to place the measurement patches in a consistent manner were always made, the corresponding topography of optodes across subjects comprising a dyad did not always correspond to the same specific location. Thus, corresponding channels did not always represent the exact same brain region across a dyad. This mismatch is a common issue in fNIRS studies. Accordingly, depending on paired (numbered) channels in dyads for performing data analyses would not necessarily establish optimal correspondence between like brain regions.

To address this issue, we sought to determine the channel pairs that represented the same brain location across members of each individual dyad. Considering the spatial resolution of the fNIRS system, we defined corresponding channels as those that were within $15 \mathrm{~mm}$ of the same brain location. We first projected the channel positions of both subjects on a common 3-D space (Figure 2). We then paired the channels of subject 1 to their closest neighboring channels of subject 2. If the closest neighboring channel was more than $15 \mathrm{~mm}$ away, they were not paired. We called channels pairs within the $15 \mathrm{~mm}$ criterion "effective channel pairs". We also determined the MNI coordinates of the middle point of each effective channel pair by using linear interpolation, and referred to that point as the "coherence channel" for the dyad.

Inter-subject coherence analysis was performed for each effective channel pair using the WTC package developed by Grinsted et al. (2004), Chang and Glover (2010) and custom MATLAB code (MathWorks). WTC is a method of measuring the cross-correlation between two time series as a function of frequency and time (Torrence and Compo, 1998), and was first applied to fNIRS hyperscanning in an earlier study from our group (Cui et al., 2012). Since then, other research studies have successfully applied WTC methodology to fNIRS data analyses (Dommer et al., 2012; Holper et al., 2012; Jiang et al., 2012, 2015). Thus, using WTC to characterize interbrain synchronization, two oxy-Hb time series were obtained

\footnotetext{
${ }^{1}$ http://www.alivelearn.net/xjview
}

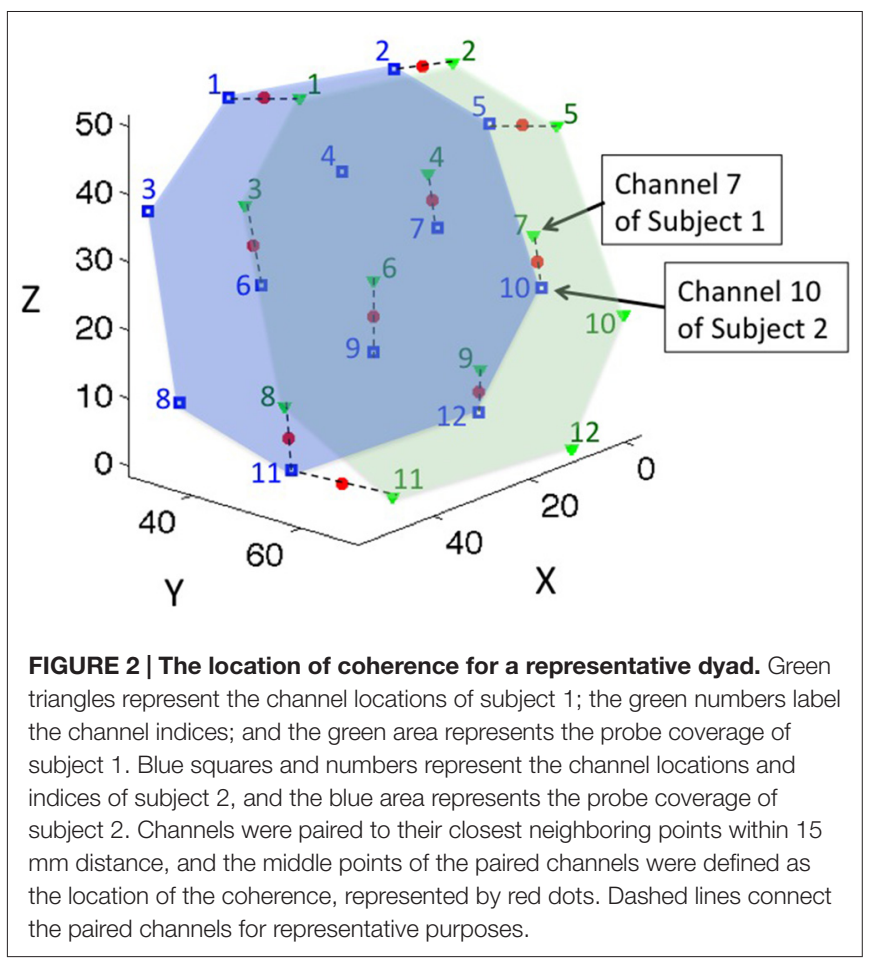

from each effective channel pair of each dyad. A frequency band was identified to further analyze the task effect. The coherence value in this band was averaged and the resulted single time series was assigned to the corresponding coherence channel.

We used a general linear model (GLM) approach to study the effect of the task. The canonical GLM was introduced by Friston et al. (1994) and has been used extensively in analysis of functional MRI, as well as fNIRS in recent years. To apply GLM, the regressors are typically convolved with a hemodynamic response function (HRF) to better mimic the brain signal. However, in this hyperscanning project, we did not study the hemodynamic response evoked by a task for a single subject. Instead, we wanted to study the coherence of two hemodynamic responses, and evaluate whether an experimental manipulation evoked meaningful activation coherence. Thus, the regressors were not convolved with any HRF. Based on the task design, we created four regressors that were based on the onset time and duration of the four conditions separately (Figure 3A). The resulting beta values at each coherence channel were used in the following group analysis to determine the task effect at a group level.

\section{Node-Wise Random-Effect Group Analysis}

For the group analysis, we developed a node-wise approach to identify common brain regions in each of the dyads, and then combined beta values from each common region into a single statistical test. Specifically, to identify common brain regions in dyads, we first generated uniformly distributed nodes on a spherical cap [Stefan Stoll, matlabCentral, stoll@phys.chem.ethz.ch], converted these nodes to MNI 


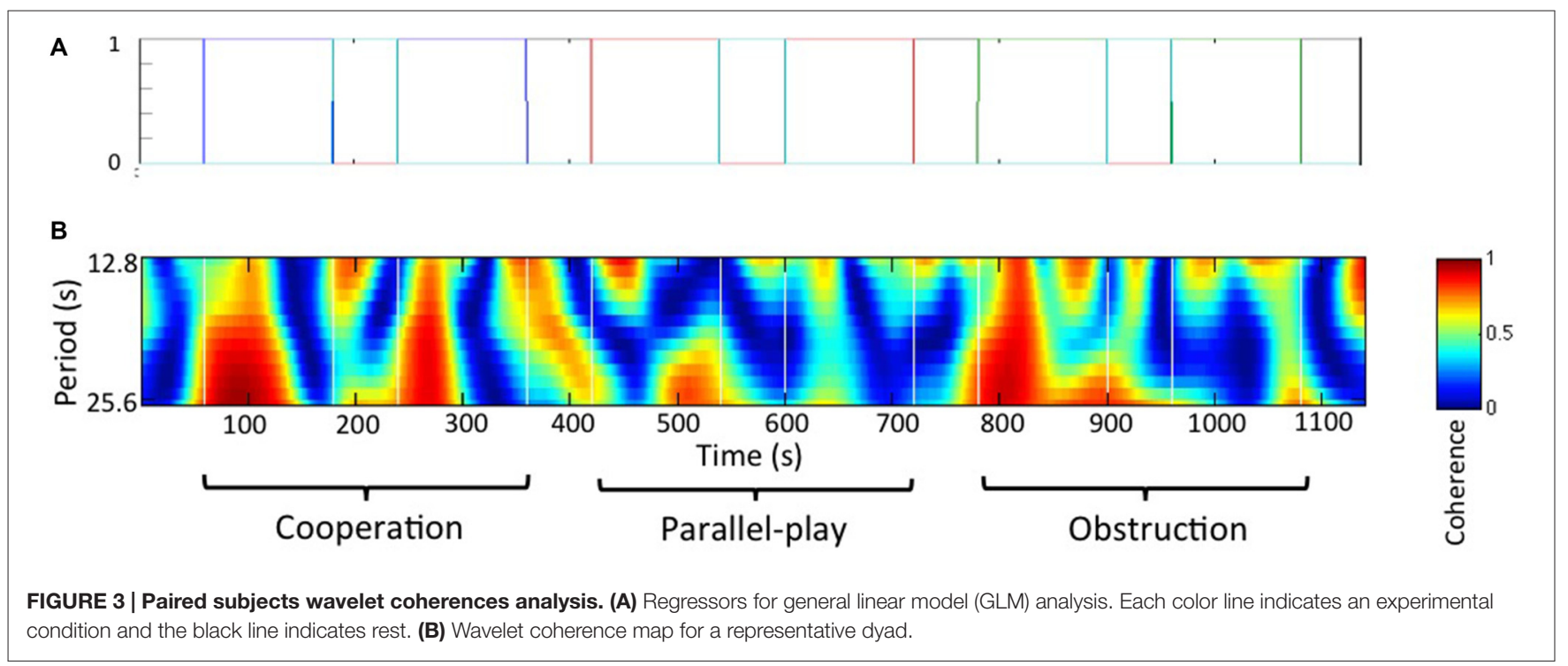

space, and projected them onto a cortical surface (Liu et al., 2015a). The generated nodes in MNI space were between 5-10 $\mathrm{mm}$ apart. We then projected all coherence channel positions from all dyads onto the same cortical surface. For each node, we counted how many dyads' coherence channels were within its $15 \mathrm{~mm}$ diameter range. Nodes were then color-coded based on the number of dyads, and were mapped onto a MRI rendered brain template in MNI space.

The statistical analysis was only performed on those nodes that had eight or nine dyads' coherence channels within their $15 \mathrm{~mm}$ diameter range. Those nodes were referred to as "effective nodes". Thus, for each effective node, there was a set of associated coherence channels. When coherence channels within a set belonged to the same dyad, we averaged the beta values to generate a single (mean) beta value for the dyad at that effective node. We then performed a onesample $t$-test of all corresponding dyads' beta values for each effective node. A relatively conservative alpha value $(P<0.01)$ was used for each condition vs. rest, corrected with the Bonferroni method for multiple comparisons, to determine the inter-subject coherence on the cortical surface at the group level.

\section{RESULTS}

\section{Task Performance}

All subjects were able to follow the instructions and maintained oral communication while playing the game. The towers remained standing during the cooperative and obstructive interaction conditions for all dyads, but fell for two subjects (two separate dyads) within the last $15 \mathrm{~s}$ of the parallel play condition. The average height of the tower at the end of the cooperation condition was $13.3 \pm 0.44 \mathrm{~cm}$, while the average height at the end of the parallel play condition was $13.7 \pm 0.86 \mathrm{~cm}$. The time intervals between two continuous movements of the

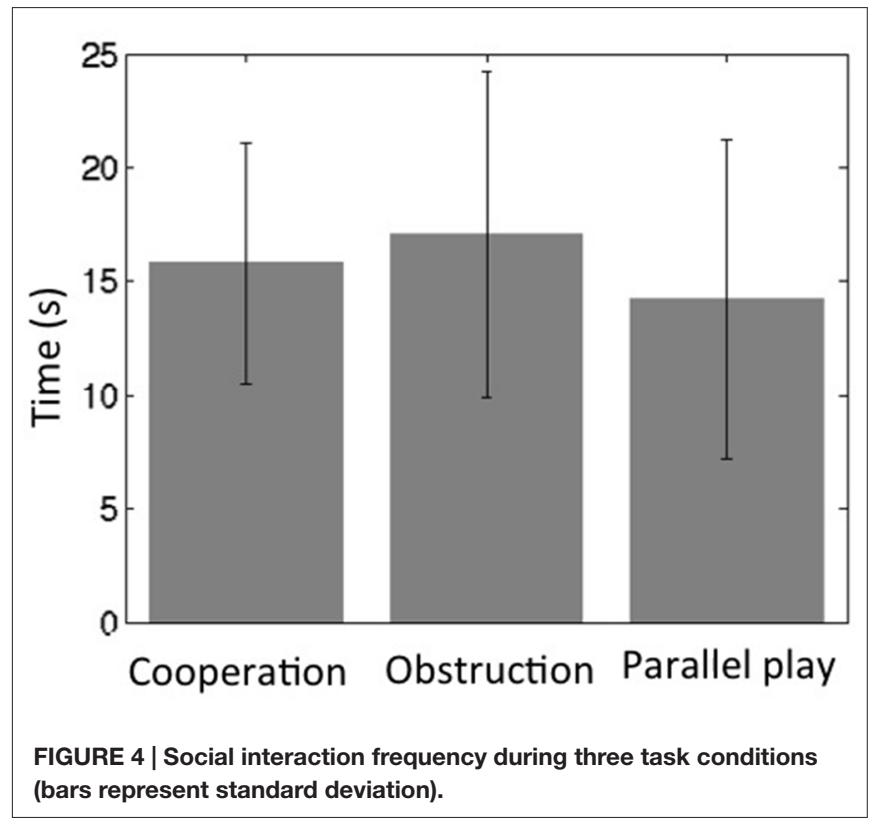

wooden block across all dyads for the cooperation condition was $16 \pm 5.3 \mathrm{~s}$, for the obstruction condition $17 \pm 7.1 \mathrm{~s}$, and for the parallel play condition $14 \pm 7.0 \mathrm{~s}$ (Figure 4). Two-sample $T$-tests revealed that there were no significant differences between the cooperation condition and the other two conditions for time interval (coop vs. obstr, $P=0.26$; coop vs. para, $P=0.12$ ). However, there was a significant time interval difference between the obstructive and parallel play conditions $(P=0.01)$.

\section{Functional Imaging}

\section{Paired Subject Wavelet Coherences}

The frequency band for the wavelet coherences analysis was determined based on social interaction frequency 

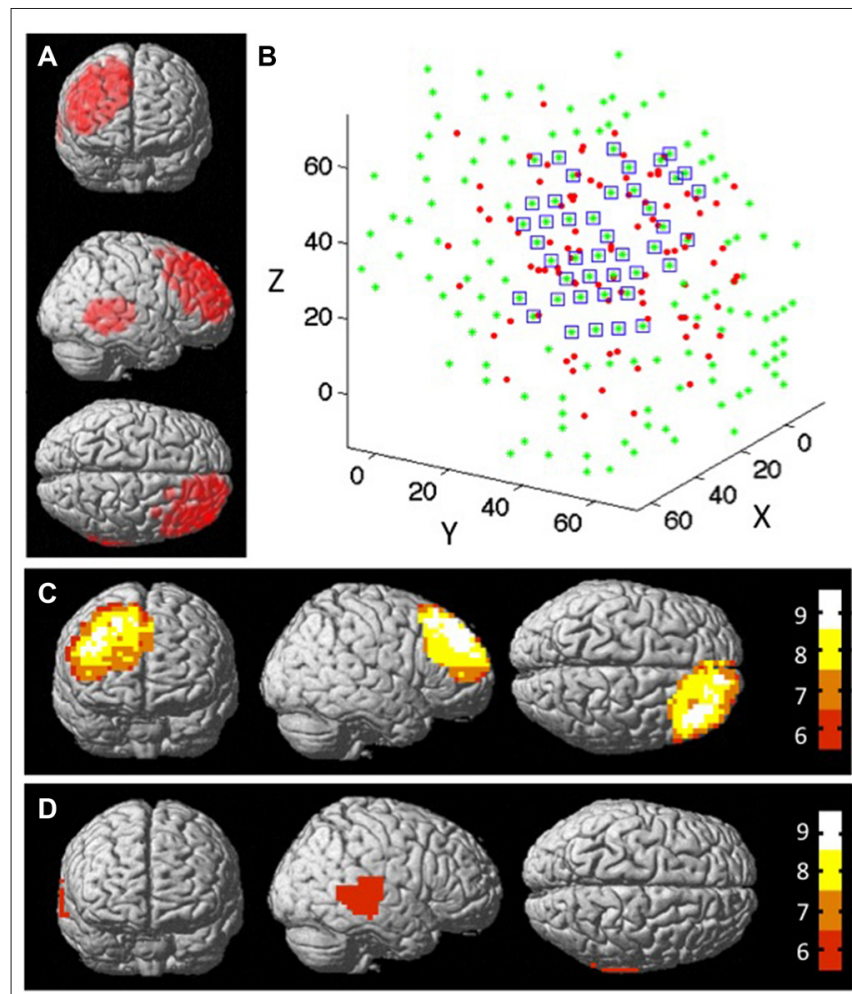

FIGURE 5 | The structural node-based spatial registration approach for inter-dyad analyses. (A) The measured channel locations of all dyads on a MRI rendered brain template. (B) The measured channel locations of all dyads at $\mathrm{rPFC}$ region in $3 \mathrm{D}$ space. The red dots represent the effective locations of all dyads; the green dots represent the nodes that cover the measured cortical region; and the blue squares mark the nodes that have data from $\geq 8$ subjects within $15 \mathrm{~mm}$ diameter distance from that node. Overlap map shows the distribution of the number of dyads that were measured by functional near-infrared spectroscopy (fNIRS): (C) at rPFC region, and (D) at rSTS region. Colorbar indicates the number of subjects that fall within $15 \mathrm{~mm}$ diameter distance from the node.

of the dyads during the task, or the inverse of the time interval between two continuous movements of the wooden block. We chose the frequency between $0.08 \mathrm{~Hz}$ to $0.04 \mathrm{~Hz}$ (period between $12.8 \mathrm{~s}$ and $25.6 \mathrm{~s}$ ) for this study based on the fact that our primary focus was on interbrain synchrony during the cooperation and obstructive conditions. Thus, this frequency band was chosen as it best corresponded to activities during these conditions. A wavelet coherence map for a representative dyad is shown in Figure 3B.

\section{Distribution of fNIRS measurement}

Figure $5 \mathbf{A}$ shows the distribution of all measured cortical locations across dyads using an MRI rendered brain template (xjView). This distribution covered the right prefrontal and right posterior temporal regions, which included the posterior portion of the rSTS. Figures 5C,D show the distribution of the number of dyads at the right prefrontal and right posterior temporal regions. The results indicate that the overlapping regions covered by at least eight dyads were mainly in the center
TABLE 1 | Foci of significant effective nodes associated with cooperative and obstructive conditions.

\begin{tabular}{|c|c|c|c|c|c|c|}
\hline \multirow[t]{2}{*}{ Region } & \multirow{2}{*}{$\begin{array}{c}\text { Brodmann } \\
\text { area }\end{array}$} & \multicolumn{3}{|c|}{ MNI coordinates } & \multirow{2}{*}{$\begin{array}{c}Z \\
\text { score }\end{array}$} & \multirow{2}{*}{$\begin{array}{c}P \text { value } \\
\text { (Bonferroni } \\
\text { corrected) }\end{array}$} \\
\hline & & $x$ & $y$ & $z$ & & \\
\hline \multicolumn{7}{|l|}{ Cooperative condition } \\
\hline Superior frontal gyrus & 8 & 22 & 48 & 46 & 2.65 & 0.0041 \\
\hline Superior frontal gyrus & 8 & 26 & 40 & 50 & 2.98 & 0.0014 \\
\hline Superior frontal gyrus & 8 & 30 & 28 & 58 & 2.94 & 0.0016 \\
\hline Superior frontal gyrus & 8 & 30 & 32 & 54 & 3.83 & 0.0001 \\
\hline Superior frontal gyrus & 8 & 34 & 24 & 58 & 2.86 & 0.0021 \\
\hline $\begin{array}{l}\text { Superior/Middle } \\
\text { frontal gyrus }\end{array}$ & 8 & 34 & 40 & 46 & 3.47 & 0.0003 \\
\hline Middle frontal gyrus & 9 & 34 & 44 & 42 & 2.80 & 0.0025 \\
\hline $\begin{array}{l}\text { Superior/Middle } \\
\text { frontal gyrus }\end{array}$ & 8 & 38 & 32 & 50 & 3.01 & 0.0013 \\
\hline Middle frontal gyrus & 8 & 38 & 36 & 46 & 2.61 & 0.0045 \\
\hline Middle frontal gyrus & 8 & 42 & 28 & 50 & 2.49 & 0.0063 \\
\hline Middle frontal gyrus & 9 & 42 & 44 & 34 & 2.38 & 0.0087 \\
\hline \multicolumn{7}{|l|}{ Obstructive condition } \\
\hline Superior frontal gyrus & 8 & 30 & 32 & 54 & 2.58 & 0.0050 \\
\hline $\begin{array}{l}\text { Superior/Middle } \\
\text { frontal gyrus }\end{array}$ & 8 & 34 & 40 & 46 & 2.42 & 0.0078 \\
\hline
\end{tabular}

of the overall measured regions. Outer edge regions were covered by less than eight dyads. The results also indicate that there were no regions in the rSTS that were covered by eight dyads' measurements (Figure 5D). The green stars in Figure 5B show the distribution of all of the nodes within the rPFC. Among them, 43 were effective nodes, marked by blue squares in the figure.

\section{Inter-Subject Cerebral Coherence During Cooperative Game Play}

Group analysis was performed only on the effective nodes covered by at least eight dyads' measurements. The rSTS region had no such effective nodes. This limited the group analysis to 43 effective nodes within the $\mathrm{rPFC}$ region and none in the STS region. We tested whether there was intersubject cerebral coherence during each task condition vs. rest. We found strong coherence during both cooperative and obstructive conditions $(P<0.01$, Bonferroni corrected), but not during the parallel play condition and the dialog section. There were 11 effective nodes associated with the cooperative condition and two effective nodes associated with the obstructive condition (Table 1, Figures 6A,B). The effective nodes associated with cooperative condition were located in the posterior region of middle and superior frontal gyrus, in particular Brodmann's Areas 8 and 9 (BA8 and BA9). The effective nodes associated with the obstructive condition overlapped with two effective nodes associated with the cooperative condition, and were located in BA8 only. Figure $6 \mathrm{C}$ shows the $T$-values at an effective node in BA8 that was significantly synchronized for both cooperative and obstructive interaction conditions. Note that the $T$-value for the cooperative condition is larger than that of the obstructive interaction condition. Figure $6 \mathrm{D}$ shows the $T$-values at an effective node in BA9 that was significantly synchronized for 

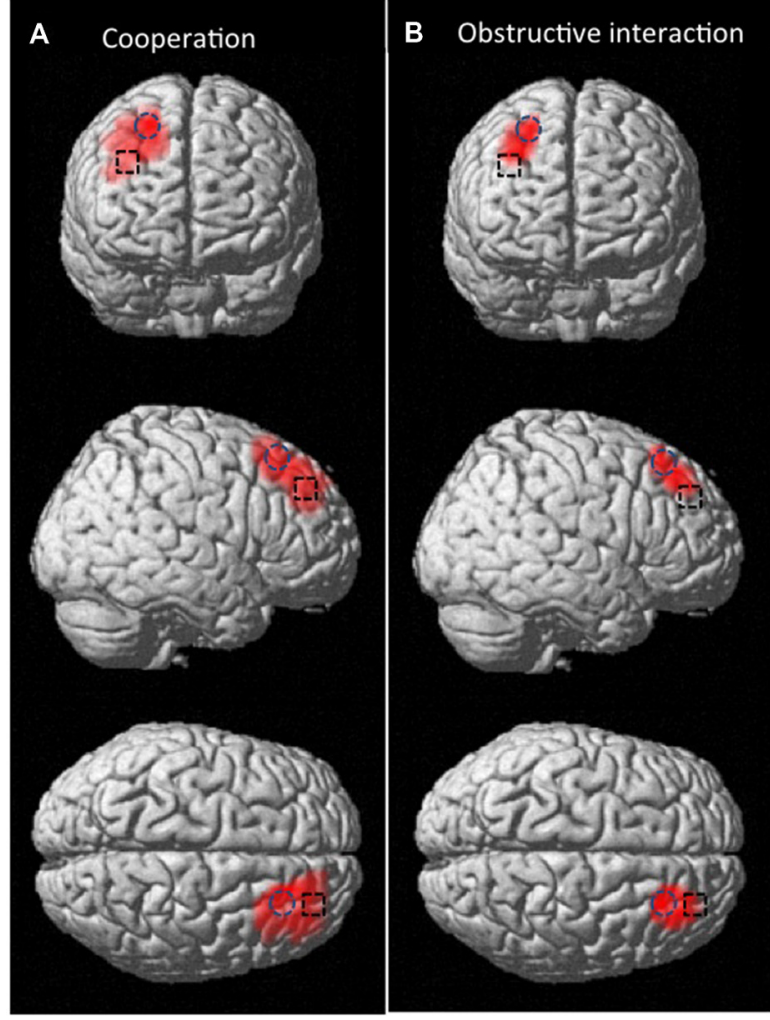

C T-value at an overlapping region, $\mathrm{MNI}$ coordinate $(30 ; 32 ; 54)$ (blue circle)

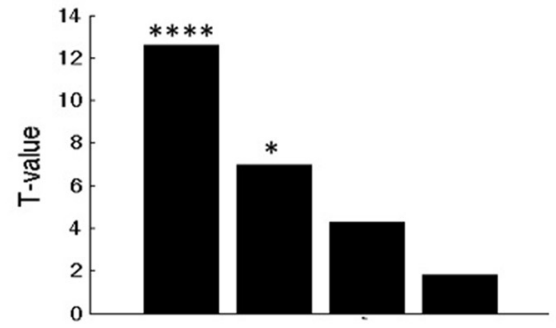

D T-value at a non-overlapping region,

MNI coordinate $(34 ; 44 ; 42)$ (black square)

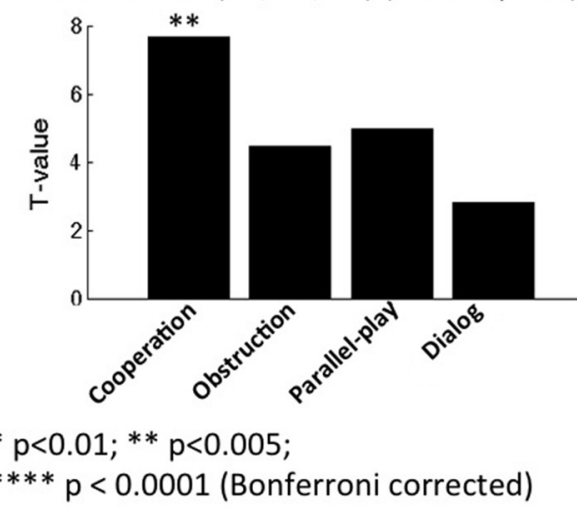

FIGURE 6 | Group t-maps for the contrast corresponding to coherence (A) in the cooperation condition vs. rest, and (B) in obstructive interaction condition vs. rest. (C) $T$-values at a node that was significantly synchronized in both cooperation and obstruction conditions (vs. rest). (D) $T$-values at a node that was significantly synchronized only in the cooperation condition (vs. rest).

the cooperative condition but not for the obstructive interaction condition.

We also tested whether there was significant inter-subject cerebral coherence difference between any two conditions. There were no nodes that showed statistically significant between-condition coherence differences at the group level. However, based on uncorrected $\mathrm{P}$ values, two regions of potential interest to human social cognition were observed (Figure 7). One region (MNI coordinates: 46, 28, 46) located at the middle frontal gyrus (BA8) showed a trend for differentiating coherence during the cooperation vs. dialog contrast (cooperation $>$ dialog, $t=3.02, P=0.017$, uncorrected; Figure 7A). Similarly, another region (MNI coordinates: 42, 52, 22 and 46, 48, 22) located at middle frontal gyrus (BA10) showed a trend for differentiating coherence during the obstructive vs. dialog contrast (obstruction $>$ dialog, [46 48 22] : $t=3.40, P=0.009$, uncorrected; [42 52 22] : $t=3.43, P=0.011$, uncorrected; Figure 7B). Both BA 8 and 10 are thought to be involved in social cognition, especially as occurring between two people (McCabe et al., 2001; Grimes, 2003; Chorvat and McCabe, 2004). Further studies with a larger sample size and appropriately corrected significance threshold are needed to further explore these findings.

\section{DISCUSSION}

It has been realized that "an important step forward in social neuroscience is the development of paradigms allowing for the study of such true, real-time social interactions" (Singer, 2012). Our study was an effort to address this limitation by establishing a novel experimental design that models naturalistic interdependent social behavior and communicative interactions between people.

All participants were able to follow the instructions based on the observation of the operators. Participants refrained from interaction during the parallel play condition and engaged in appropriate interaction during other conditions. The average time interval between two continuous movements of the wooden block was longer for the cooperation and obstruction conditions than for parallel play condition. This indicates that the participants understood the instructions and implemented some form of interaction during the appropriate conditions.

Strong inter-brain synchrony was observed at two effective nodes in the middle and superior frontal gyrus (BA8) during the cooperative and obstructive interaction conditions, but not during the parallel play condition and the dialog section. This finding suggests that BA8 cortical regions were involved in goal-oriented social interaction in our experiment. Studies 


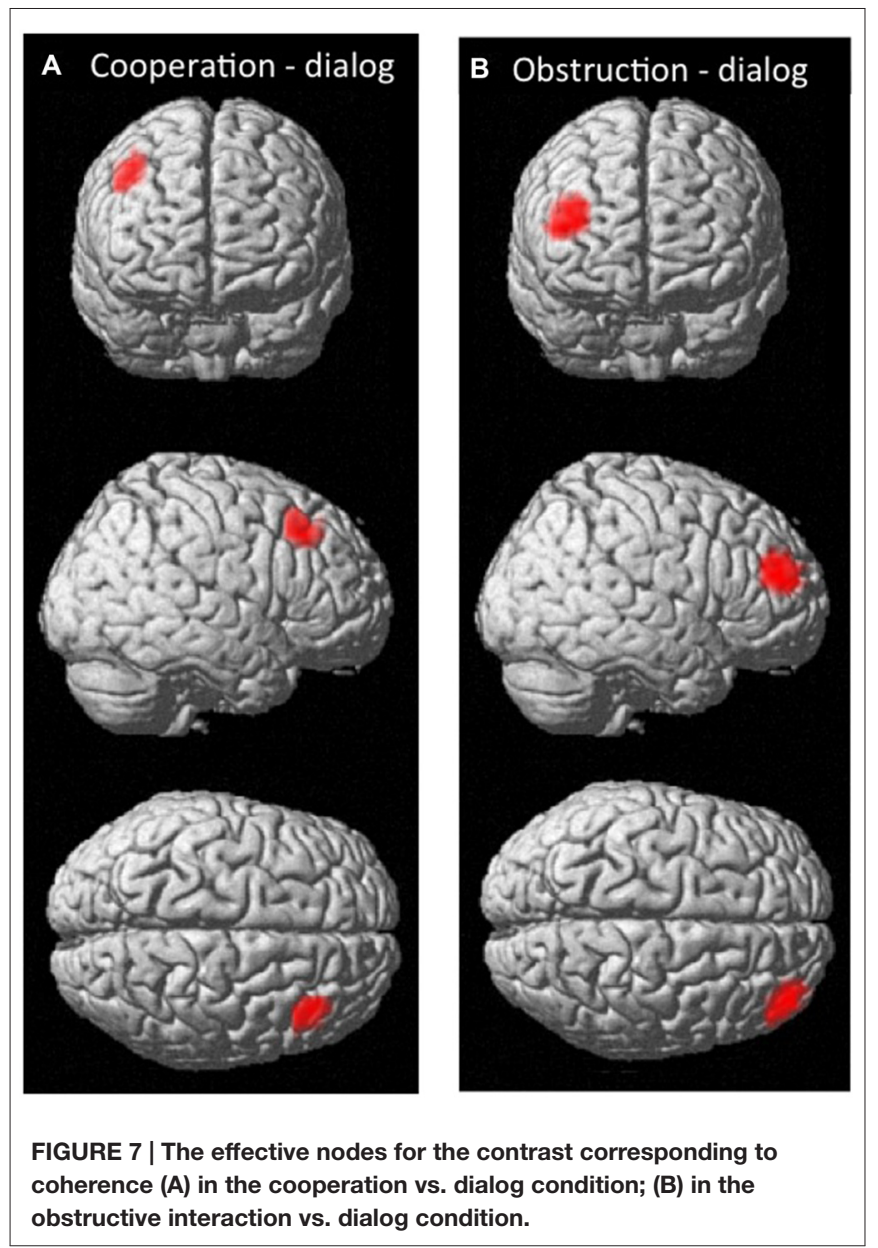

have been shown that BA8 is active when individuals plan complex movements, make decisions that are uncertain, and use prospective memory to remember to do something in the future (Okuda et al., 1998; Fincham et al., 2002; Volz et al., 2005). In our turn-based study, individuals in the dyad were continuously making decisions based on their partner's suggestions and responses during the cooperation and obstructive conditions, but not during the parallel play condition. That is to say, our results suggest that the observed coherence in this area might be a feature of social decision-making when two people interact. A recent study by Tang et al. (2016) investigated the INS in the right dorsolateral prefrontal cortex (rDLPFC) during an economic exchange task that involved oral communication within dyads. They used the channel-wise data analysis method, and the results indicated increased (but not significant) synchronization in rDLPFC channels (Figure 3C in Tang et al., 2016). The difference between Tang's results and the results reported here might indicate that BA8 was involved in complex interactive movements but not pure dyadic communication in social cognition.

Inter-brain synchrony was observed at nine additional effective nodes in dorsomedial prefrontal cortex (dmPFC; BA9) only during the cooperative interaction condition. The $\mathrm{dmPFC}$ is thought to be part of the brain network that is activated by considering the intentions of another individual in social processing (Behrens et al., 2008, 2009). This region has been demonstrated to be active during "theory of mind" (ToM) games played with other individuals, but not played with computers (Greene and Haidt, 2002; Amodio and Frith, 2006; Saxe, 2006). During the cooperative condition in our version of the Jenga game, two players were instructed to communicate and agree on each movement before they physically moved the wooden block, which meant they had to fully understand their partner in order to reach the goal of building the wooden tower as tall as possible. This interaction was associated with increased inter-brain synchronization. However, in the obstructive condition, participants may have been less likely to fully regard or consider their partner's recommendations when making their final decision on block movement. When combined with previous findings, our results suggest the involvement of dmPFC (BA9) in cooperative social behavior.

It is noteworthy that the coherence region associated with cooperation (covered by 11 effective nodes total) was much larger than that associated with the obstructive interaction (covered by two effective nodes). This indicates that cooperative interaction recruited more prefrontal regions than the obstructive interaction in this turn-based Jenga game. This finding might also be associated with task difficulty in that the cooperation condition might have been harder than the obstruction condition.

In this study we also developed a method to identify synchronized channels within a dyad and a structural node-based spatial registration approach for inter-dyad analyses. Compared with existing methods, this new approach does not require a structural MRI for each participant, does not need interpolation, and allows for preservation of the spatial resolution of the fNIRS imaging system. More importantly, the approach described here can help to offset the inevitable variability in fNIRS probe placement on an individual subject's head, making it possible to combine data from multiple dyads to a common stereotaxic space for group analyses.

There were several limitations to our study. First, our sample size was small, and we were able to cover only a portion of the brain given the number of channels available with our fNIRS instrument. Therefore, important parts of the rSTS might not have been captured for some dyads, and we were unable to perform statistical analysis for that region. Additionally, during the group analysis, we performed a Bonferroni correction based on $n=43$ (total number of effective nodes). However, based on our definition of an effective node there was likely inter-node (spatial) dependence and thus, the method of correction based on the number of nodes may have been too conservative by overestimating the number of independent spatial units. Third, we only videotaped five dyads, which limited our ability to analyze behavioral data and assess associations between these data and fNIRS activation. Fourth, the choice of values for the upper and lower limits of the frequency band for the WTC analysis was relatively arbitrary. Future research could focus on a more quantitative method to determining these values. 


\section{CONCLUSION}

In summary, the present study reveals inter-brain neural synchronization in the prefrontal cortex, in particular BA8 and BA9, during a naturally occurring Jenga game with faceto-face communication. Inter-brain synchrony was observed in BA8 during both cooperative and obstructive interaction conditions but not during the parallel play condition and the dialog section. These results indicate that BA8 plays a role in goal-oriented social interactions such as complex interactive movements and social decision-making. Inter-brain synchrony was also observed in BA9 during the cooperative interaction condition but not during the obstructive interaction and the parallel play conditions. These additional findings suggest that BA9 may be particularly engaged when ToM is required for cooperative social interaction. In addition, we also developed a method to identify synchronized channels for each dyad

\section{REFERENCES}

Allison, T., Puce, A., and McCarthy, G. (2000). Social perception from visual cues: role of the STS region. Trends Cogn. Sci. 7, 267-278. doi: 10.1016/s13646613(00)01501-1

Amodio, D. M., and Frith, C. D. (2006). Meeting of minds: the medial frontal cortex and social cognition. Nat. Rev. Neurosci. 7, 268-277. doi: 10. 1038/nrn1884

Astolfi, L., Cincotti, F., Mattia, D., De Vico Fallani, F., Salinari, S., Marciani, M. G., et al. (2009). Estimation of the cortical activity from simultaneous multi-subject recordings during the prisoner's dilemma. Conf. Proc. IEEE Eng. Med. Biol. Soc. 2009, 1937-1939. doi: 10.1109/IEMBS.2009.5333456

Babiloni, F., and Astolfi, L. (2014). Social neuroscience and hyperscanning techniques: past, present and future. Neurosci. Biobehav. Rev. 44, 76-93. doi: 10. 1016/j.neubiorev.2012.07.006

Babiloni, C., Vecchio, F., Infarinato, F., Buffo, P., Marzano, N., Spada, D., et al. (2011). Simultaneous recording of electroencephalographic data in musicians playing in ensemble. Cortex 47, 1082-1090. doi: 10.1016/j.cortex.2011.05.006

Baess, P., Zhdanov, A., Mandel, A., Parkkonen, L., Hirvenkari, L., Mäkelä, J. P., et al. (2012). MEG dual scanning: a procedure to study real-time auditory interaction between two persons. Front. Hum. Neurosci. 6:83. doi: 10 . 3389/fnhum.2012.00083

Balconi, M., and Molteni, E. (2015). Past and future of near-infrared spectroscopy in studies of emotion and social neuroscience. J. Cogn. Psychol. 28, 129-146. doi: 10.1080/20445911.2015.1102919

Behrens, T. E., Hunt, L. T., and Rushworth, M. F. (2009). The computation of social behavior. Science 324, 1160-1164. doi: 10.1126/science.1169694

Behrens, T. E., Hunt, L. T., Woolrich, M. W., and Rushworth, M. F. (2008). Associative learning of social value. Nature 456, 245-249. doi: 10. 1038/nature07538

Briggs, K. M., Runyon, M. K., and Deblinger, E. (2011). “The use of play in traumafocused cognitive-behavioral therapy," in Play in Clinical Practice: EvidenceBased Approaches, eds S. W. Russ and L. N. Niec (New York, NY: Guilford Press), 168-200.

Chang, C., and Glover, G. H. (2010). Time-frequency dynamics of resting-state brain connectivity measured with fMRI. Neuroimage 50, 81-98. doi: 10.1016/j. neuroimage.2009.12.011

Chorvat, T., and McCabe, K. (2004). The brain and the law. Philos. Trans. R. Soc. Lond. B Biol. Sci. 359, 1727-1736. doi: 10.1098/rstb.2004.1545

Cui, X., Bray, S., Bryant, D. M., Glover, G. H., and Reiss, A. L. (2011). A quantitative comparison of NIRS and fMRI across multiple cognitive tasks. Neuroimage 54, 2808-2821. doi: 10.1016/j.neuroimage.2010.10.069

Cui, X., Bryant, D. M., and Reiss, A. L. (2012). NIRS-based hyperscanning reveals increased interpersonal coherence in superior frontal cortex during and a structural node-based spatial registration approach for inter-dyad analysis. These novel methods have the potential to significantly extend fNIRS hyperscanning applications to social cognitive research.

\section{AUTHOR CONTRIBUTIONS}

NL, CM, EEW and AHP: study design, collect data, data analysis, manuscript preparation. JEC: data analysis, manuscript preparation. ALR: study design, manuscript preparation.

\section{ACKNOWLEDGMENTS}

A grant from the NIH to ALR (S10 RR024657) and the Albert $\mathrm{Yu}$ and Mary Bechmann Foundation provided financial support for this study. We also thank Dr. Gary Glover for his helpful comments on task design and data analysis.

cooperation. Neuroimage 59, 2430-2437. doi: 10.1016/j.neuroimage.2011. 09.003

Delpy, D. T., Cope, M., van der Zee, P., Arridge, S., Wray, S., and Wyatt, J. (1988). Estimation of optical pathlength through tissue from direct time of flight measurement. Phys. Med. Biol. 33, 1433-1442. doi: 10.1088/00319155/33/12/008

Dommer, L., Jäger, N., Scholkmann, F., Wolf, M., and Holper, L. (2012). Betweenbrain coherence during joint $n$-back task performance: a two-person functional near-infrared spectroscopy study. Behav. Brain Res. 234, 212-222. doi: 10. 1016/j.bbr.2012.06.024

Duane, T. D., and Behrendt, T. (1965). Extrasensory electroencephalographic induction between identical twins. Science 150:367. doi: 10.1126/science.150. 3694.367

Fincham, J. M., Carter, C. S., van Veen, V., Stenger, V. A., and Anderson, J. R. (2002). Neural mechanisms of planning: a computational analysis using eventrelated fMRI. Proc. Natl. Acad. Sci. U S A 99, 3346-3351. doi: 10.1073/pnas. 052703399

Friston, K. J., Holmes, A. P., Worsley, K. J., Poline, J. P., Frith, C. D., and Frackowiak, R. S. (1994). Statistical parametric maps in functional imaging: a general linear approach. Hum. Brain Mapp. 2, 189-210. doi: 10.1002/hbm. 460020402

Funane, T., Kiguchi, M., Atsumori, H., Sato, H., Kubota, K., and Koizumi, H. (2011). Synchronous activity of two people's prefrontal cortices during a cooperative task measured by simultaneous near-infrared spectroscopy. J. Biomed. Opt. 16:077011. doi: 10.1117/1.3602853

Greene, J., and Haidt, J. (2002). How (and where) does moral judgment work? Trends Cogn. Sci. 6, 517-523. doi: 10.1016/s1364-6613(02) 02011-9

Grimes, K. (2003). To trust is human. New Scientist 178, 32-37.

Grinsted, A., Moore, J. C., and Jevrejeva, S. (2004). Application of the cross wavelet transform and wavelet coherence to geophysical time series. Nonlinear Process Geophys. 11, 561-566. doi: 10.5194/npg-11-561-2004

Hari, R., and Kujala, M. V. (2009). Brain basis of human social interaction: from concepts to brain imaging. Physiol. Rev. 89, 453-479. doi: 10.1152/physrev. 00041.2007

Holper, L., Scholkmann, F., and Wolf, M. (2012). Between-brain connectivity during imitation measured by fNIRS. Neuroimage 63, 212-222. doi: 10.1016/j. neuroimage.2012.06.028

Jiang, J., Chen, C., Dai, B., Shi, G., Ding, G., Liu, L., et al. (2015). Leader emergence through interpersonal neural synchronization. Proc. Natl. Acad. Sci. U S A 112, 4274-4279. doi: 10.1073/pnas.1422930112

Jiang, J., Dai, B., Peng, D., Zhu, C., Liu, L., and Lu, C. (2012). Neural synchronization during face-to-face communication. J. Neurosci. 32, 16064-16069. doi: 10.1523/JNEUROSCI.2926-12.2012 
Kawaguchi, H., Murakami, B., and Kawai, M. (2010). Behavioral characteristics of children with high functioning pervasive developmental disorders during a game. J. Epidemiol. 20, S490-S497. doi: 10.2188/jea.je20090178

Kawasaki, M., Yamada, Y., Ushiku, Y., Miyauchi, E., and Yamaguchi, Y. (2013). Inter-brain synchronization during coordination of speech rhythm in humanto-human social interaction. Sci. Rep. 3:1692. doi: 10.1038/srep01692

Liu, N., Cui, X., Bryant, D. M., Glover, G. H., and Reiss, A. L. (2015a). Inferring deep-brain activity from cortical activity using functional nearinfrared spectroscopy. Biomed. Opt. Express 6, 1074-1089. doi: 10.1364/BOE. 6.001074

Liu, T., Saito, H., and Oi, M. (2015b). Role of the right inferior frontal gyrus in turn-based cooperation and competition. Brain Cogn. 99, 17-23. doi: 10.1016/j. bandc.2015.07.001

Liu, T., Saito, H., and Oi, M. (2015c). Obstruction increases activation in the right inferior frontal gyrus. Soc. Neurosci. 14, 1-9. doi: 10.1080/17470919.2015. 1088469

Liu, T., and Pelowski, M. (2014). Clarifying the interaction types in two-person neuroscience research. Front. Hum. Neurosci. 8:276. doi: 10.3389/fnhum.2014. 00276

McCabe, K., Houser, D., Ryan, L., Smith, V., and Trouard, T. (2001). A functional imaging study of cooperation in two-person reciprocal exchange. Proc. Natl. Acad. Sci. U S A 98, 11832-11835. doi: 10.1073/pnas.211415698

Montague, P. R., Berns, G. S., Cohen, J. D., McClure, S. M., Pagnoni, G., Dhamala, M., et al. (2002). Hyperscanning: simultaneous fMRI during linked social interactions. Neuroimage 164, 1159-1164. doi: 10.1006/nimg.2002. 1150

Okamoto, M., Dan, H., Shimizu, K., Takeo, K., Amita, T., Oda, I., et al. (2004). Multimodal assessment of cortical activation during apple peeling by NIRS and fMRI. Neuroimage 21, 1275-1288. doi: 10.1016/j.neuroimage.2003. 12.003

Okamoto, M., Tsuzuki, D., Clowney, L., Dan, H., Singh, A. K., and Dan, I. (2009). Structural atlas-based spatial registration for functional near-infrared spectroscopy enabling inter-study data integration. Clin. Neurophysiol. 120, 1320-1328. doi: 10.1016/j.clinph.2009.01.023

Okuda, J., Fujii, T., Yamadori, A., Kawashima, R., Tsukiura, T., Fukatsu, R., et al. (1998). Participation of the prefrontal cortices in prospective memory: evidence from a PET study in humans. Neurosci. Lett. 253, 127-130. doi: 10.1016/s03043940(98)00628-4

Page-Gould, E., Mendoza-Denton, R., and Tropp, L. R. (2008). With a little help from my cross-group friend: reducing anxiety in intergroup contexts through cross-group friendship. J. Pers. Soc. Psychol. 95, 1080-1094. doi: 10.1037/00223514.95.5.1080

Paulson, F. L. (1974). Teaching cooperation on television. AV Commun. Rev. 22, $229-246$.

Saxe, R. (2006). Uniquely human social cognition. Curr. Opin. Neurobiol. 16, 235-239. doi: 10.1016/j.conb.2006.03.001
Scholkmann, F., Holper, L., Wolf, U., and Wolf, M. (2013). A new methodical approach in neuroscience: assessing inter-personal brain coupling using functional near-infrared imaging (fNIRS) hyperscanning. Front. Hum. Neurosci. 7:813. doi: 10.3389/fnhum.2013.00813

Singer, T. (2012). The past, present and future of social neuroscience: a European perspective. Neuroimage 61, 437-449. doi: 10.1016/j.neuroimage.2012.01.109

Singh, A. K., Okamoto, M., Dan, H., Jurcak, V., and Dan, I. (2005). Spatial registration of multichannel multi-subject fNIRS data to MNI space without MRI. Neuroimage 27, 842-851. doi: 10.1016/j.neuroimage.2005.05.019

Tang, H., Mai, X., Wang, S., Zhu, C., Krueger, F., and Liu, C. (2016). Interpersonal brain synchronization in the right temporo-parietal junction during faceto-face economic exchange. Soc. Cogn. Affect. Neurosci. 11, 23-32. doi: 10. $1093 /$ scan/nsv092

Torrence, C., and Compo, G. P. (1998). A practical guide to wavelet analysis. Bull. Am. Meteorol. Soc. 79, 61-78. doi: 10.1175/15200477(1998)079\%3C0061:APGTWA\%3E2.0.CO;2

Tsuzuki, D., Cai, D. S., Dan, H., Kyutoku, Y., Fujita, A., Watanabe, E., et al. (2012). Stable and convenient spatial registration of stand-alone NIRS data through anchor-based probabilistic registration. Neurosci. Res. 72, 163-171. doi: 10. 1016/j.neures.2011.10.008

Tsuzuki, D., and Dan, I. (2014). Spatial registration for functional nearinfrared spectroscopy: from channel position on the scalp to cortical location in individual and group analyses. Neuroimage 85, 92-103. doi: 10.1016/j. neuroimage.2013.07.025

Volz, K. G., Schubotz, R. I., and von Cramon, D. Y. (2005). Variants of uncertainty in decision-making and their neural correlates. Brain Res. Bull. 67, 403-412. doi: 10.1016/j.brainresbull.2005.06.011

Wright, S. C., Aron, A., and Tropp, L. R. (2002). "Including others (and groups) in the self: self-expansion and intergroup relations," in The Social Self: Cognitive, Interpersonal and Intergroup Perspectives, eds J. P. Forgas and K. D. Williams (Philadelphia: Psychology Press), 343-363.

Yanagisawa, H., Dan, I., Tsuzuki, D., Kato, M., Okamoto, M., Kyutoku, Y., et al. (2010). Acute moderate exercise elicits increased dorsolateral prefrontal activation and improves cognitive performance with stroop test. Neuroimage 50, 1702-1710. doi: 10.1016/j.neuroimage.2009.12.023

Conflict of Interest Statement: The authors declare that the research was conducted in the absence of any commercial or financial relationships that could be construed as a potential conflict of interest.

Copyright (C) 2016 Liu, Mok, Witt, Pradhan, Chen and Reiss. This is an open-access article distributed under the terms of the Creative Commons Attribution License (CC $B Y)$. The use, distribution and reproduction in other forums is permitted, provided the original author(s) or licensor are credited and that the original publication in this journal is cited, in accordance with accepted academic practice. No use, distribution or reproduction is permitted which does not comply with these terms. 